\title{
Brain strain rate response: addressing computational ambiguity and experimental data for
}

\section{model validation}

Zhou Zhou, ${ }^{1 *}$ Xiaogai Li, ${ }^{1}$ Yuzhe Liu, ${ }^{2,3}$ Warren N. Hardy, ${ }^{4}$ Svein Kleiven ${ }^{1}$

${ }^{1}$ Neuronic Engineering, KTH Royal Institute of Technology, Stockholm, 14152, Sweden.

${ }^{2}$ Department of Bioengineering, Stanford University, Stanford, CA, 94305, USA.

${ }^{3}$ School of Biological Science and Medical Engineering, Beihang University, Beijing 100083, China.

${ }^{4}$ Virginia Tech-Wake Forest Center for Injury Biomechanics, Blacksburg, VA, 24061, USA. 


\section{Highlights}

- A theoretical clarification of two strain rate computational schemes was presented, in which the strain rate was calculated as the time derivative of strain or derived from the strain-rate tensor.

- Discordant cross-scheme responses were substantiated by experimental principal strain rate and computational principal and tract-oriented strain rates.

- Cross-scheme comparison of strain rate responses is inappropriate.

- The newly calculated experimental strain rate curves can be used for strain rate validation of FE head model 


\section{Abstract}

Traumatic brain injury (TBI) is an alarming global public health issue with high morbidity and mortality rates. Although the causal link between external insults and consequent brain injury remains largely elusive, both strain and strain rate are generally recognized as crucial factors for brain injury development. With respect to the flourishment of strain-based exploration, concerning ambiguity and inconsistency are noted in the scheme for strain rate calculation within the TBI research community. Furthermore, there is no experimental data that can be used to validate the strain rate responses of finite element (FE) models of the human brain. Thus, the current work presented a theoretical clarification of two commonly used strain rate computational schemes: the strain rate was either calculated as the time derivative of strain or derived from the strain-rate tensor. To further substantiate this theoretical disparity, these two schemes were respectively implemented to estimate the strain rate responses from a previous-published cadaveric experiment and an FE brain model secondary to a concussive impact. The results clearly showed scheme-dependent responses, both in the experimentally determined principal strain rate and FE-derived principal and tract-oriented strain rates. The results suggest that cross-scheme comparison of strain rate responses is inappropriate, and the utilized strain rate computational scheme needs to be reported in future studies. The newly calculated experimental strain rate curves can be used for strain rate validation of FE head models.

\section{Keywords}

Traumatic brain injury; time derivative of strain; strain-rate tensor; strain rate validation 


\section{Graphic abstract}

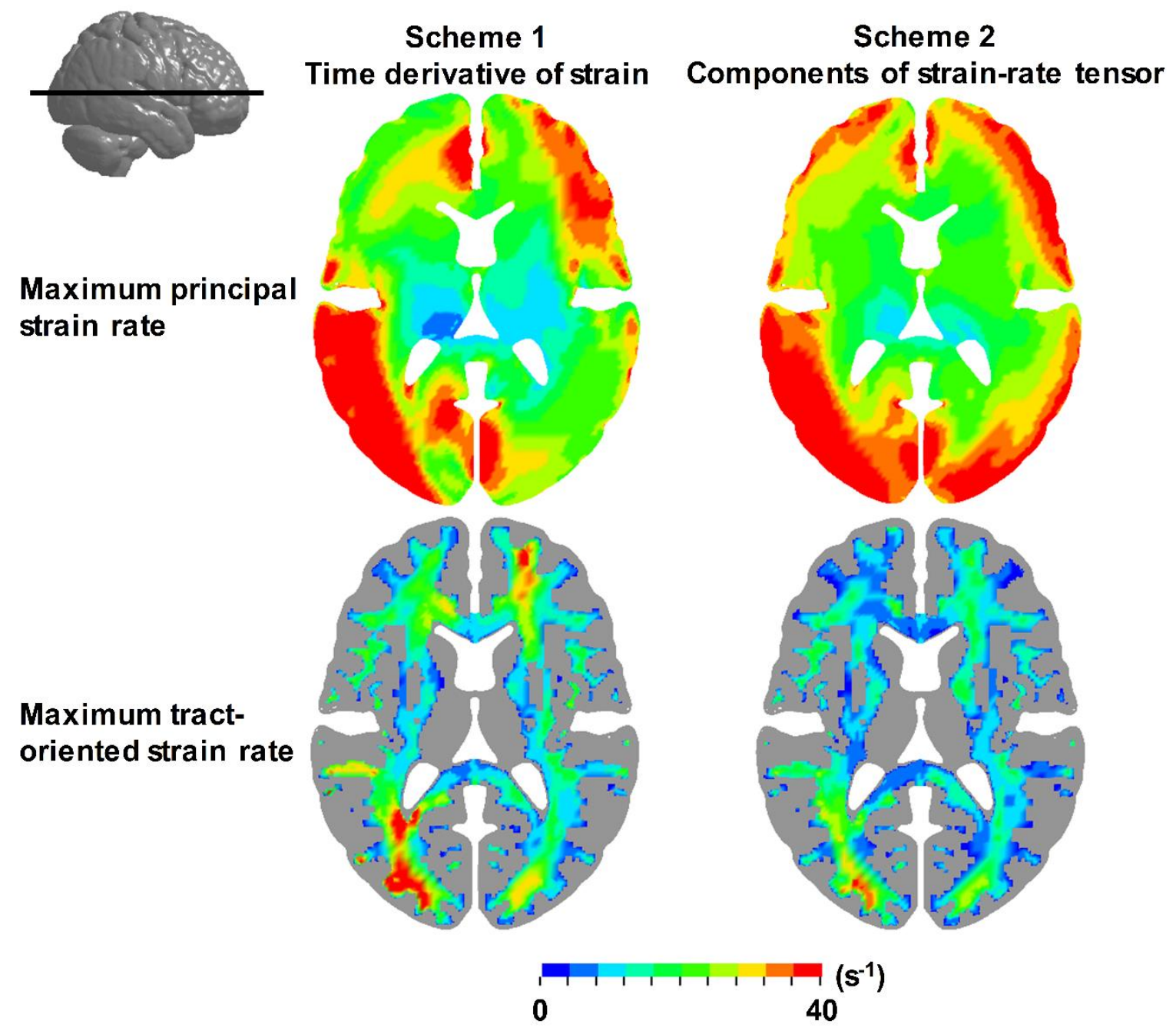

Contour maps of maximum principal strain rate and maximum tract-oriented strain rate computed by scheme 1 (i.e., time derivate of strain) and scheme 2 (i.e., components of strain rate tensor), respectively. 


\section{Introduction}

Traumatic brain injury (TBI), defined as an alternation in brain function or other evidence of brain pathology caused by an external force [1], is a major global public health threat. Recent epidemiological studies estimated that in the United States, around 61000 people died from TBIinduced injuries in 2019 [2], and in the European Union, there were approximately 82000 TBIassociated deaths in 2012 [3]. Despite collective efforts from the scientific community, clinical services, and policymakers to prevent the occurrence and mitigate the detriment of TBI, no clear improvement has been noted over the past two decades [4]. Efforts to better understand brain injury pathogenesis and develop more effective head protective strategies are vital to resolve this concerning epidemic.

Although the mechanobiological pathway from external insult, localized tissue responses, and resultant brain injury remains to be better explored, both strain and strain rate have been generally recognized as crucial factors for brain injury development [5]. For example, through relating controlled mechanical inputs to ensuing neurite morphology in a three-dimensional (3D) in vitro compression model, Bar-Kochba, et al. [6] found that the localization of neurite blebbing correlated with shear strain along the axial direction of neurons, while neurite swelling was highly loading-rate dependent. Montanino, et al. [7] utilized a composite finite element (FE) model of a single axon to predict the responses of axonal subcomponents secondary to tensile elongation, revealing a dependency of axolemma deformation on both the magnitude and the rate of imposed loadings. These experimental and computational findings collectively underscored the importance of monitoring both the strain and strain rate during head impacts.

In light of this, various strain-based metrics have been proposed over the past decades, such as maximum principal strain (MPS) in the brain $[8,9]$, cumulative strain damage measure (CSDM) quantifying the volume fraction of brain experiencing strain levels over a pre-defined threshold [10, 11], maximum tract-oriented strain (MTOS) measuring the stretch along axonal fiber tract [12, 13]. Many studies employed these strain-based metrics as measures to describe brain responses during 
head impacts. To date, strain-based measurements have been experimentally quantified by Hardy, et al. [14] (recently reanalysed by Zhou, et al. [15]) based on MPS (0.07-0.22) in cadaveric experiments with loading conditions close to traumatic levels, and by Knutsen, et al. [16] based on MPS (0.02-0.05) and MTOS (0.005-0.03) in voluntary loading scenarios. These strain-based metrics were also used to establish kinematic-based brain injury risk functions with the aid of FE head models. For example, the Brain Injury Criteria (BrIC) [17] was proposed based on the dependency of MPS and CSDM on the angular velocity observed from two FE models of human brain $[10,18]$. Discussions are ongoing to potentially adopt this newly proposed BrIC into the New Car Assessment Program in the vehicle safety standards [19].

Compared with the many strain-based studies, there are fewer investigations on brain strain rate. This is evidenced by the large body of literature that merely examined the strain-based outputs (e.g., the studies by Zhou, et al. [20] and Bian and Mao [9]). For those investigations with a focus on strain rate, it is ubiquitous that the strain rate values are directly reported without any clarification on how they are calculated (e.g., the studies by Beckwith, et al. [21] and Post, et al. [22]). Even when limiting to the handful of studies with the strain rate computational schemes elaborated, alarming inconsistency existed. In two experimental paradigms, Rashid, et al. [23] determined the strain rate from the symmetric part of the velocity gradient in the simple shear experiments of brain tissue, while LaPlaca and Thibault [24] quantified strain rate as the time derivative of strain in an in vitro TBI system. In the computational works by Zhou, et al. [25] and Carlsen, et al. [26], the strain rate was derived from the strain-rate tensor, while King, et al. [27] instead calculated the strain rate by differentiating the FEderived maximum principal strain vs. time curves. Concerningly, little or limited knowledge exists on how this disparity in computational schemes affects the resultant strain rate values.

When scrutinizing the existing strain rate-relevant investigations employing FE models of human head, it is common that the model is validated against the brain-skull relative motion and/or brain strain and then directly used for strain rate prediction. As highlighted by Zhou, et al. [28], if the model is evaluated against an experimental parameter that is not the parameter of interest itself, it is 
suspected that numerical error might be introduced and propagated through the chain of calculation from the evaluated parameter to the parameter of interest (e.g., the relationship between brain motion/strain and brain strain rate). Thus, direct evaluation of brain strain rate response of the FE model is preferred. Unfortunately, there is no existing experimental brain strain rate dataset with loading conditions close to traumatic levels. The reliability of the FE-derived strain rate responses remains to be appropriately evaluated.

The current study endeavoured to address the ambiguity relevant to the calculation of brain strain rate and provide experimental data for strain rate evaluation of FE models of the human brain. Thus, a theoretical clarification of two currently used strain rate computational schemes was presented, in which the strain rates were computed as the time derivatives of strains of interest, and the first eigenvalues or components of interest of the strain-rate tensor, respectively. These two schemes were then implemented to calculate the experimental principal strain rate and computational principal and tract-oriented strain rates, through which the distinction between these two schemes was substantiated. The newly calculated experimental strain rate curves can be served as a reference to evaluate the strain rate responses of FE models.

\section{Methods}

\subsection{Theoretical basis}

This section presents a continuum mechanical description of the two prevalent strain rate computational schemes (referred to as scheme 1 and scheme 2 hereafter), in which the calculation of two specific strain rates are elaborated (i.e., the first principal strain rate and the tract-oriented strain rate). Thus, two strain rates in combination with two computation schemes resulted in a total of 4 types of strain rates, all of which are used as response variables in TBI studies. The mathematical symbols used throughout the text are clarified below: MPSR and MPSR represent the maximum 
first principal strain rates computed by scheme 1 and scheme 2, respectively; MTOSR and MTOSR respectively denote the maximum tract-oriented strain rates following scheme 1 and scheme 2.

As is the common context of these two schemes, the motion $\varphi(\mathbf{X}, t)$ maps the position vector of every point in the brain in the undeformed configuration ( $\mathbf{X}$ ) (i.e., $\mathbf{X}=\left\{\begin{array}{lll}X_{1} & X_{2} & X_{3}\end{array}\right\}$ ) to its deformed configuration ( $\mathbf{x}$ ) at time $\mathrm{t}$ (i.e., $\left.\mathbf{x}(t)=\left\{x_{1}(t) \quad x_{2}(t) \quad x_{3}(t)\right\}\right)$. The temporal derivative of $\boldsymbol{\varphi}(\mathbf{X}, t)$ at fixed position $\mathbf{X}$ represents the velocity, writing as $\mathbf{v}(\mathbf{X}, t)=\left\{\begin{array}{lll}v_{1}(t) & v_{2}(t) \quad v_{3}(t)\end{array}\right\}$.

\subsubsection{Scheme 1: Time derivative of strain}

Scheme 1 calculates the strain rates as the time derivatives of the first eigenvalue or the component of interest of the strain tensor $\mathbf{E}(t)$. Given that $\boldsymbol{\varphi}(\mathbf{X}, t)$ consists of both deformation and rigid-body motion, the deformation gradient $\mathbf{F}(t)$ is introduced, isolating the deformation through mapping line elements $(d \mathbf{X})$ in the reference configuration into its counterpart $(d \mathbf{x})$ in the current configuration.

$$
\mathbf{F}(t)=\frac{\partial \boldsymbol{\varphi}(\mathbf{X}, t)}{\partial \mathbf{X}}=\left[\begin{array}{ccc}
\frac{\partial x_{1}(t)}{\partial X_{1}} & \frac{\partial x_{1}(t)}{\partial X_{2}} & \frac{\partial x_{1}(t)}{\partial X_{3}} \\
\frac{\partial x_{2}(t)}{\partial X_{1}} & \frac{\partial x_{2}(t)}{\partial X_{2}} & \frac{\partial x_{2}(t)}{\partial X_{3}} \\
\frac{\partial x_{3}(t)}{\partial X_{1}} & \frac{\partial x_{3}(t)}{\partial X_{2}} & \frac{\partial x_{3}(t)}{\partial X_{3}}
\end{array}\right]
$$

The Green-Lagrange strain tensor is then calculated as

$$
\mathbf{E}(t)=\frac{1}{2}\left(\mathbf{F}^{\mathrm{T}}(t) \mathbf{F}(t)-\mathbf{I}\right)
$$

where the subscript T represents the transpose operation and $\mathbf{I}$ is the identify tensor. 
The first principal strain ( $\operatorname{PS}(t))$ is computed as the maximum eigenvalue of $\mathbf{E}(t)$ and the tract-oriented strain ( $\operatorname{TOS}(t))$ is obtained by projecting $\mathbf{E}(t)$ along the real-time fiber orientation $(\mathbf{a}(t))$ (equation 3) [13]:

$$
\operatorname{TOS}(t)=\mathbf{a}^{\mathrm{T}}(t) \cdot \mathbf{E}(t) \cdot \mathbf{a}(t)
$$

Resultantly, the first principal strain rate $(\operatorname{PSR}(t))$ and tract-oriented strain rate $(\operatorname{TOSR}(t))$ were computed as the derivative of $\operatorname{PS}(t)$ and $\operatorname{TOS}(t)$ with respect to time (equation 4 and 5 ), respectively. Of note, a five-point stencil neural network derivative function was used for all the derivative operations in the current study following previous studies [29-31].

$$
\begin{aligned}
\operatorname{PSR}(t) & =\frac{\partial \mathrm{PS}(t)}{\partial t} \\
\operatorname{TOSR}(t) & =\frac{\partial \operatorname{TOS}(t)}{\partial t}
\end{aligned}
$$

The maximum value of $\operatorname{PSR}(t)$ and $\operatorname{TOSR}(t)$ across the simulated time domain was identified (i.e., MPSR and MTOSR, respectively). Scheme 1 has been used in previous TBI studies to extract the maximum principal strain rate [27, 29-39] and maximum tract-oriented strain rate [29, 30, 32-36, 38], respectively.

\subsubsection{Scheme 2: Strain-rate tensor}

Scheme 2 calculates strain rate as the first eigenvalue or the component of interest of the strain-rate tensor $\mathbf{d}(t)$. Of note, the strain-rate tensor is synonymous with rate of deformation tensor [40]. In contrast with the deformation gradient $\mathbf{F}(t)$ based on displacement field, velocity gradient $\mathbf{l}(t)$ is derived from velocity field $\mathbf{v}(\mathbf{X}, t)$ by taking its spatial gradient according to equation 6 : 


$$
\mathbf{l}(t)=\frac{\partial \mathbf{v}(\mathbf{X}, t)}{\partial \mathbf{x}}=\left[\begin{array}{ccc}
\frac{\partial v_{1}(t)}{\partial x_{1}} & \frac{\partial v_{1}(t)}{\partial x_{2}} & \frac{\partial v_{1}(t)}{\partial x_{3}} \\
\frac{\partial v_{2}(t)}{\partial x_{1}} & \frac{\partial v_{2}(t)}{\partial x_{2}} & \frac{\partial v_{2}(t)}{\partial x_{3}} \\
\frac{\partial v_{3}(t)}{\partial x_{1}} & \frac{\partial v_{3}(t)}{\partial x_{2}} & \frac{\partial v_{3}(t)}{\partial x_{3}}
\end{array}\right]
$$

The symmetrical part of $\mathbf{I}(t)$ is defined as the strain-rate tensor $\mathbf{d}(t)$ :

$$
\mathbf{d}(t)=\frac{1}{2}\left(\mathbf{l}(t)+\mathbf{l}^{\mathrm{T}}(t)\right)
$$

The first principal strain rate $(\hat{\operatorname{PSR}}(t))$ and tract-oriented strain rate $(\hat{\operatorname{TOSR}}(t))$ were respectively computed as the maximum eigenvalue of $\mathbf{d}(t)$ and the projection of $\mathbf{d}(t)$ along the realtime fiber orientation ( $\mathbf{a}(t)$ ) (equation 8).

$$
\hat{\operatorname{TOSR}}(t)=\mathbf{a}^{\mathrm{T}}(t) \cdot \mathbf{d}(t) \cdot \mathbf{a}(t)
$$

The time-accumulated peaks of $\hat{\operatorname{PSR}}(t)$ and $\hat{\operatorname{TOSR}}(t)$ were determined and labelled as $\hat{\mathrm{MPSR}}$ and MTOSR, respectively. Scheme 2 has been previously used to extract the principal strain rate and/or the tract-oriented strain rates in the brain $[23,25,26,41-44]$ and other biological tissues (e.g., leg muscles [45-48], tongue [49, 50], myocardium [51-53]).

While collectively considering these two schemes, it is worth mentioning that the material time derivative of the Green-Lagrange strain tensor (i.e., $\mathbf{E}$ in scheme 1) is the pull-back of the strainrate tensor (i.e., $\mathbf{d}$ in scheme 2) (equation 9) [40, 54]. Note that $\dot{\mathbf{E}}$ in equation 9 represents the time derivative of $\mathbf{E}$.

$$
\dot{\mathbf{E}}=\mathbf{F}^{\mathrm{T}} \cdot \mathbf{d} \cdot \mathbf{F}
$$

\subsection{Experimental strain rate}


To substantiate the disparity between scheme 1 and scheme 2 from an experimental perspective and provide experimental data for strain rate evaluation of FE brain model, the principal strain rates in the cadaveric experiments were respectively computed using both schemes. In Hardy, et al. [14], thirty-five impacts were conducted on eight inverted and perfused head-neck complexes of post-mortem human subjects, in which the motion of the radiopaque neutral density targets (NDTs) implanted within the cadaveric brain was measured using a high-speed, bi-planar X-ray system with a temporal resolution of $1 \mathrm{~ms}$. To expedite the estimation of local responses, Hardy, et al. [14] deployed 7 NDTs in a cluster configuration with the center target being $10 \mathrm{~mm}$ from the resting six counterparts, roughly occupying $1.5 \mathrm{ml}$ of tissue. As represented by $\mathrm{C} 1$ and $\mathrm{C} 2$ in Figure $1 \mathrm{~A}$, two NDT clusters were implanted within each cadaveric brain.

Following the selection criteria proposed by Zhou, et al. [28], 15 NDT clusters from 14 impacts with both impact kinematics and motions of all 7 associated NDTs measured reliably were chosen to calculate the principal strain rates. As illustrated by a representative cluster in Figure 1B, representative FE nodes were generated based on the NDT starting positions. Based on the NDT deploying characteristics in Hardy, et al. [14], 8 tetrahedron elements were developed for each cluster by connecting each NDT to its neighbouring counterparts [15]. The tracked motions were used to excite the tetrahedron model, which was solved by the LS-DYNA software (Livermore Software Technology Corporation, LSTC). From the completed simulation, the first principal strain rates were respectively extracted as the time derivatives of the first principal strain (i.e., $\operatorname{PSR}(t)$ for the scheme 1 ) and maximum eigenvalue of the strain-rate tensor (i.e., $\hat{\operatorname{PSR}}(t)$ for scheme 2 ). The strain rates of all available tetrahedron elements were averaged to provide a general estimation of the strain rate within the volume occupied by the NDT cluster. The maximum values of $\operatorname{P\operatorname {SR}}(t)$ and $\hat{\operatorname{PSR}}(t)$ were identified (i.e., MPSR and MPSR ). Due to the unavailability of fiber orientation information of these cadavers, it is infeasible to compute tract-oriented strain rate from this experimental data. 

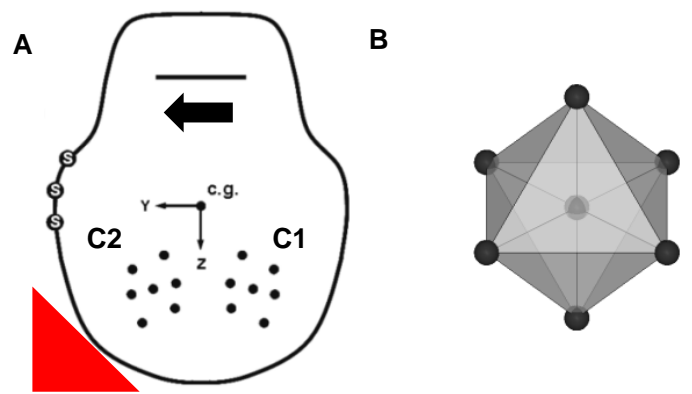

Figure 1. (A) Representative sketch of NDT cluster implanting schemes. For a given head, two NDT clusters were implanted as cluster 1 (C1) and cluster 2 (C2). (B) Strain-rate estimation with the NDTs as black spheres while tetrahedron elements in grey and mesh line in black. To visualize the center NDT, all tetrahedron elements are shown in translucency.

\subsection{Computational strain rates}

\subsubsection{The ADAPT model}

We next implemented scheme 1 and scheme 2 to respectively derive the principal strain rate and tract-oriented strain rate from an anatomically detailed head model (i.e., the ADAPT model in Figure 2). The ADAPT model was developed previously at KTH Royal Institute of Technology in Stockholm using the LS-DYNA software. Details about the model discretization and material properties are available in Li, et al. [55]. Briefly, the model includes the brain, skull, meninges (i.e., dura mater, pia mater, falx, and tentorium), subarachnoid cerebrospinal fluid (CSF), ventricle, and sinuses (i.e., superior sagittal sinus and transverse sinus). As is the structure of interest in the current study, the brain was further grouped with explicit representations of various components, including four lobes of the cerebral gray matter (GM) (Figure 2A), cerebellar GM, cerebral white matter (WM), cerebellar WM, and brainstem (Figure 2B). A second-order Ogden hyperelastic constitutive model combined with sixorder Prony series was employed to describe the nonlinearity and viscoelasticity of brain material [56]. The biofidelity of the model has been evaluated against experimental data of intracranial pressure [57], brain-skull relative displacement [14], and brain strain [15]. The strain rate responses of the model were additionally evaluated by the newly calculated experimental strain rate data in Appendix A.

To extract tract-related responses, it is imperative to monitor the real-time direction of fiber tracts during the impact [13]. Thus, the primary orientation of the WM fiber tracts was extracted from the ICBM DTI-81 atlas [58] and then embedded within the WM elements in the form of truss elements 
[13]. Through this, potential variation in fiber orientation during the impacts was reflected by the temporal direction of the truss element, which was updated at each solution cycle of the timemarching simulation. These temporally dynamic fiber orientation informed the computation of tractoriented strain rate.

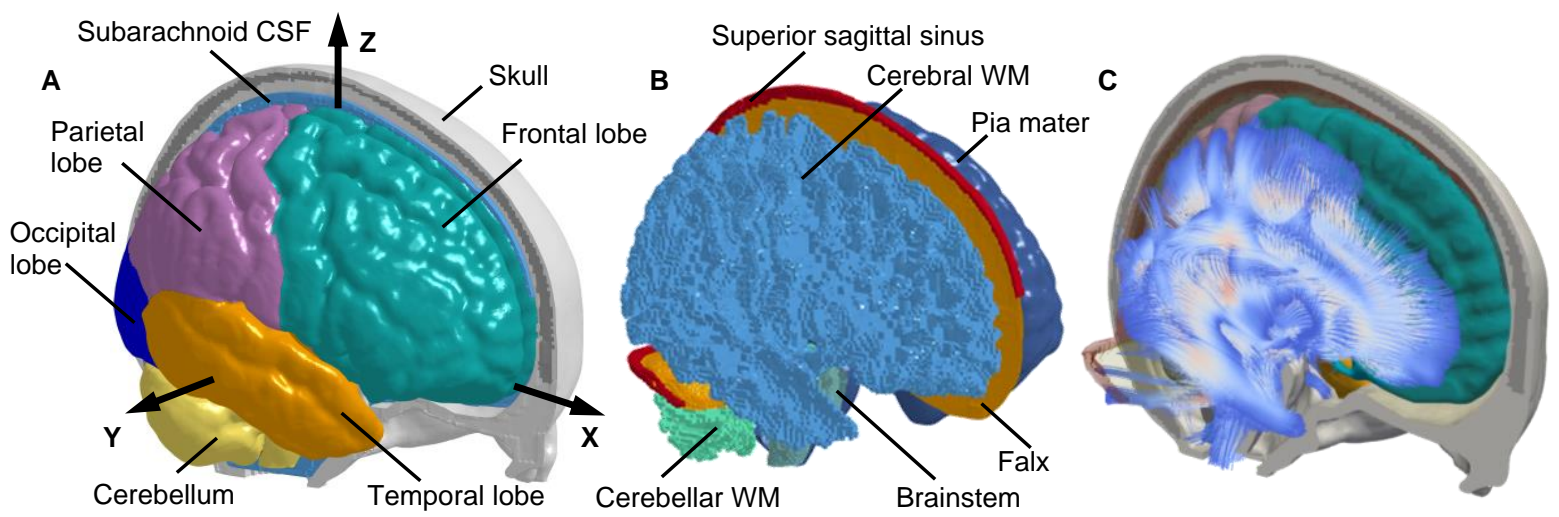

Figure 2. Finite element model of the human head with embedded truss elements. (A) Finite element model of the human head with the skull open to expose the subarachnoid cerebrospinal fluid (CSF) and brain. A skull-fixed coordinate system and corresponding axes are illustrated with the origin at the head's centre of gravity. (B) Brain components of cerebral white matter (WM), cerebellar WM, brainstem, pia mater, falx, tentorium, superior sagittal sinus, and transverse sinus. (C) Brain with fiber tracts.

\subsubsection{Loading condition and computation of principal and tract-oriented strain rates}

The ADAPT model was employed to simulate a concussive impact, through which both the maximum principal strain rates and maximum tract-oriented strain rates were respectively computed using scheme 1 and scheme 2 . The impact loading was measured by instrumented mouthguard from a collegiate American football player with self-reported post-concussive symptoms [59]. Both translational and rotational acceleration profiles (Figure 3) were applied to a node located at the head's centre of gravity and constrained to the rigidly modelled skull. The model responses were output at every $0.25 \mathrm{msec}$. 

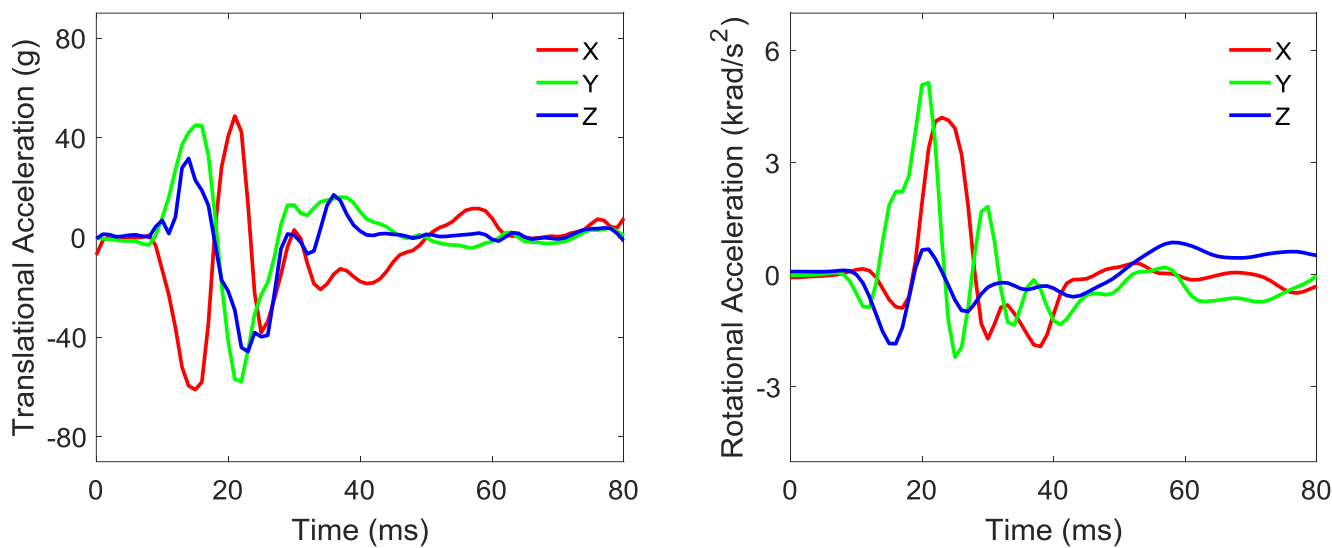

Figure 3. Head model loading conditions for one concussive impact with the kinematics measured from instrumented mouthguard. Note the $X, Y$, and $Z$ axes are the same as those in the skull-fixed coordinate system in Figure $1 \mathrm{~A}$.

Once the simulation was complete, the strain tensor and strain-rate tensor of all brain elements and the temporal orientation of the truss elements embedded within the WM elements were obtained. For each brain element, the first principal strain rates were calculated as the time derivatives of the first principal strain (i.e., scheme 1) and maximum eigenvalue of the strain-rate tensor (i.e., scheme 2) with their maximum values across the whole impact reported (i.e., MPSR and MPSR ). The tract-oriented strain rates were additionally computed for all WM elements. In scheme 1 , the strain tensor of each WM element was iteratively resolved along the temporal direction of its embedded truss element (i.e., equation 3) with the outcome being discretely differentiated between time points to obtain the tract-oriented strain rate (i.e., equation 5). In scheme 2 , the strain-rate tensor of each WM element was projected along the real-time direction of its embedded truss element (i.e., equation 8). For both schemes, the maximum values of the tract-oriented strain rates across the whole impact were reported, i.e., MTÖSR and MTÓSR .

\subsubsection{Data analysis}

To quantify the cross-scheme disparity, relative differences in strain rate peaks computed by scheme 1 and scheme 2 were computed for all brain elements based on the principal measures and for all WM elements based on the tract-oriented measures, respectively. To test whether cross-scheme strain rate results were readily translatable, linear regression analyses were conducted between 
MPSR and MPSR, and between MTOSR and MTOSR, respectively. In addition to the correlation coefficient ( $r$ ), the root-mean-square error normalized by the mean value of strain rate peaks calculated by scheme 1 (i.e., NRMSE) was reported. The threshold for significance was $p<0.05$.

\section{Results}

\subsection{Experimental principal strain rates}

Experimental principal strain rates computed by both schemes for all the 15 clusters are depicted in Figure 4. Using scheme 1, the time history curves of the principal strain rate were generally biphasic with both positive and negative phases, while the curves were monophasic with purely positive values when using scheme 2 . The strain rate responses of all 15 clusters attained the peak values within the pulse durations except for those in C241-T5 C1 and C393-T1 C1 solved by scheme 2 . A cluster-wise comparison of maximum experimental principal strain rates for the resting 13 clusters is shown in Figure 5. The maximum values ranged from $9.3 \mathrm{~s}^{-1}$ (C241-T6 C2) to $25.8 \mathrm{~s}^{-1}$ (C380-T4 C1) in scheme 1 and spanned from $15.6 \mathrm{~s}^{-1}$ (C380-T2 C1) to $35.6 \mathrm{~s}^{-1}$ (C064-T1 C1) in scheme 2. 

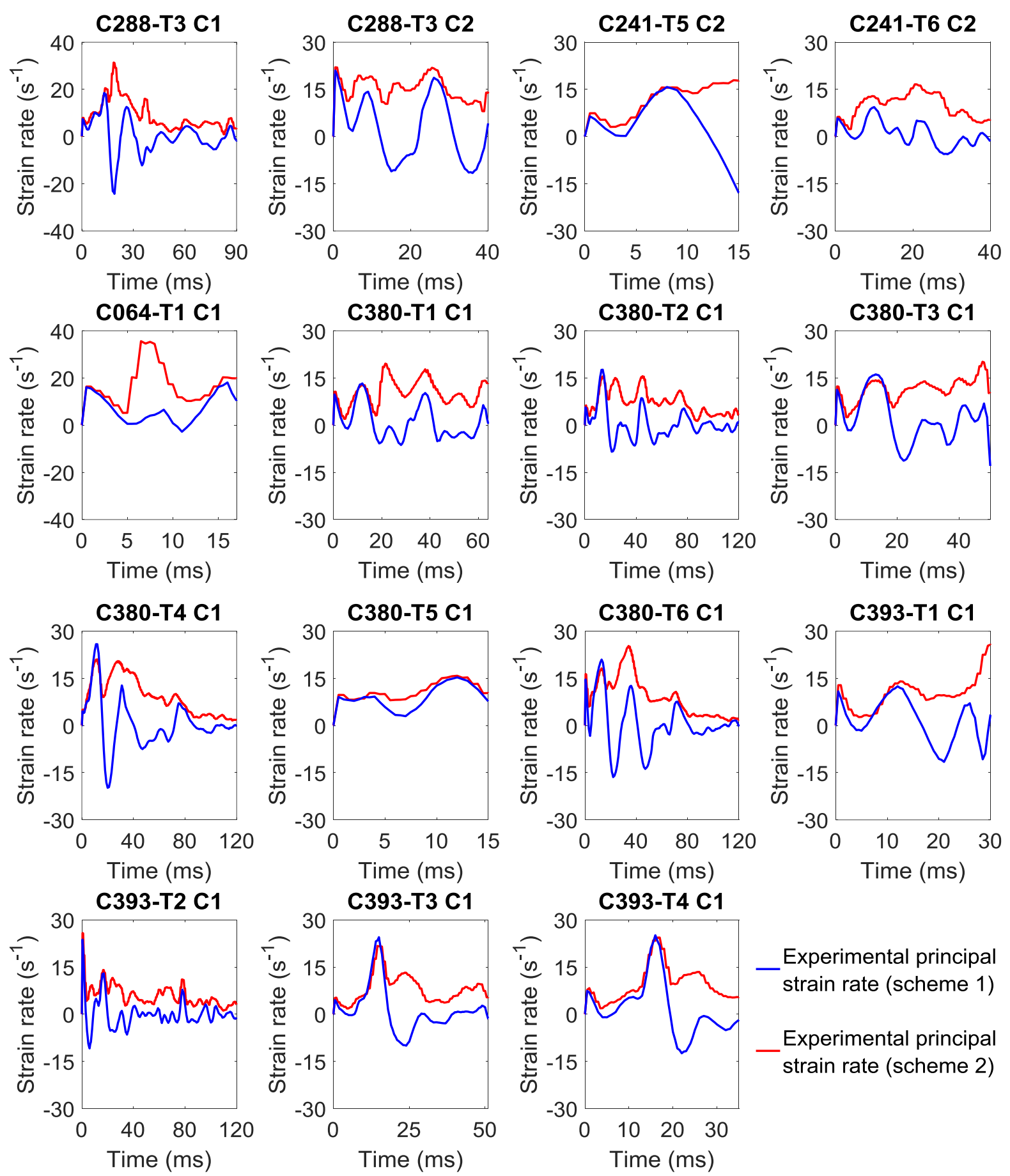

Figure 4. Comparison of experimental principal strain rates computed as time derivate of first principal strain (scheme 1) and first eigenvalue of strain-rate tensor (scheme 2). 


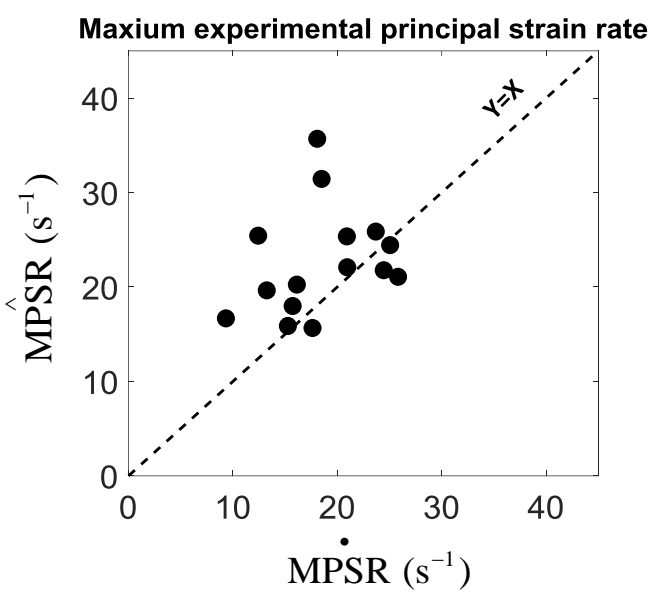

Figure 5. Cluster-wise comparison of maximum experimental principal strain rates from 13 NDT cluster, each of which the strain rates was computed by schemes 1 ( $\stackrel{\bullet}{\mathrm{MPR}}$ ) and scheme 2 (MPSR ), respectively.

\subsection{Computational principal and tract-oriented strain rates}

Figure 6A-F visually compares the maximum principal and tract-oriented strain rates at a representative horizontal cut-section. For the maximum principal strain rate (Figure $6 \mathrm{~A}-\mathrm{C}$ ), although both schemes predicted high strain rate peaks (i.e., peaks over or approaching $40 \mathrm{~s}^{-1}$ ) at the cortical region, substantial differences (i.e., differences over $10 \mathrm{~s}^{-1}$ or less than $-10 \mathrm{~s}^{-1}$ ) between MPSR and MPSR were noted in the left frontal lobe, right occipital lobe, and paraventricular region. For example, MPSR was larger than MPSR in the left temporal lobe (solid arrow in Figure $6 \mathrm{C}$ ), while a reverse trend was noted in the left frontal cortex (dash arrow in Figure 6C). When switching to maximum tract-oriented strain rate (Figure 6D-F), MTOSR and MTOSR differed in magnitude and spatial distribution. In particular, scheme 1 revealed substantially larger tract-oriented strain rate peaks than scheme 2 across the whole WM in the frontal region (solid arrows in Figure 6F), while MTOSR was relatively smaller than MTOSR in the external capsules (dash arrows in Figure 6F).

This visible difference was further quantified by linear regression analysis between two schemes (Figure 6G-H). Although a significant correlation $(p<0.001)$ was attained for both types of strain rates, the correlation coefficients $(r)$ were 0.82 for maximum principal strain rate and 0.77 for maximum tract-oriented strain rate. Across the whole brain elements, a NRMSE value of $16.69 \%$ was 
computed between MPSR and MPSR, while across the whole WM elements, a NRMSE value of 27.35\% was calculated between MTÓSR and MTÓSR .
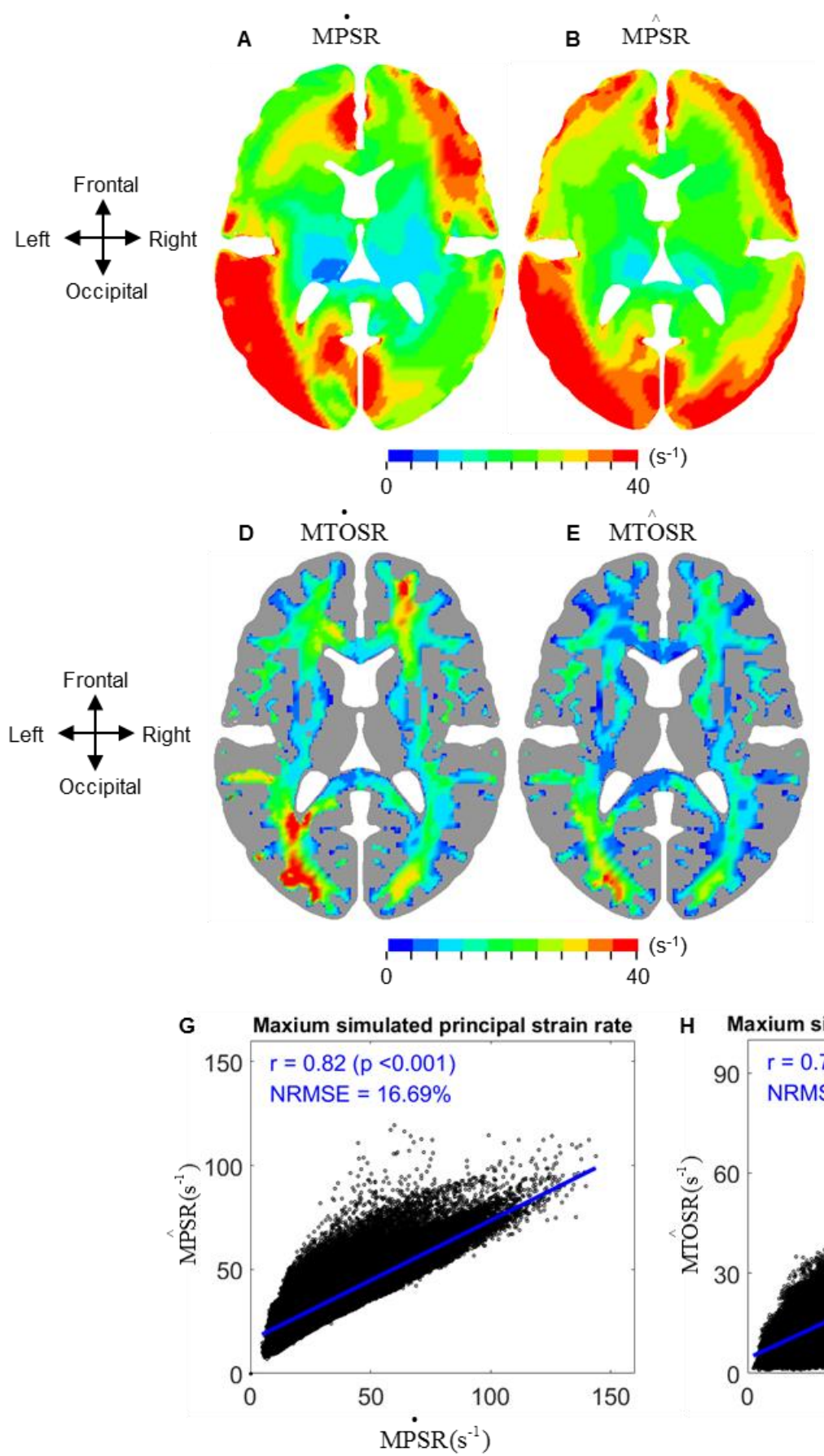

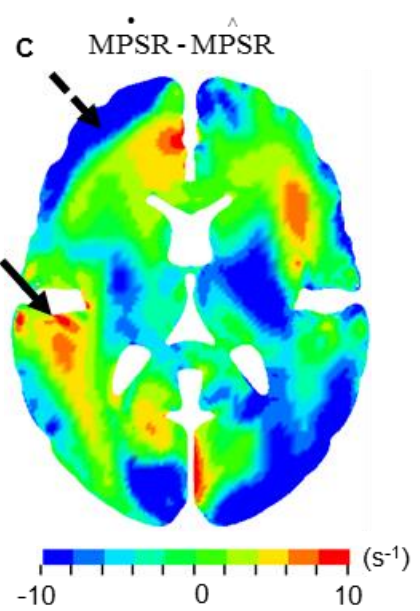

F MTÓSR-MTÓSR

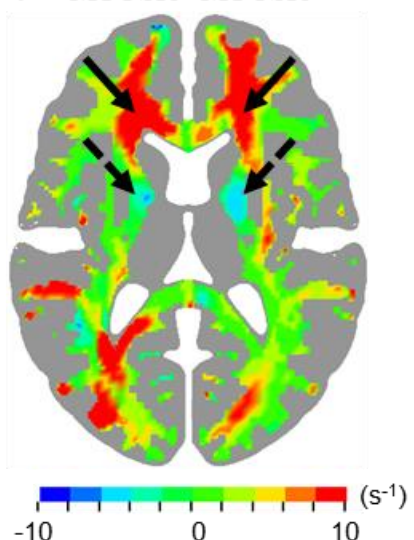

$-10$

10

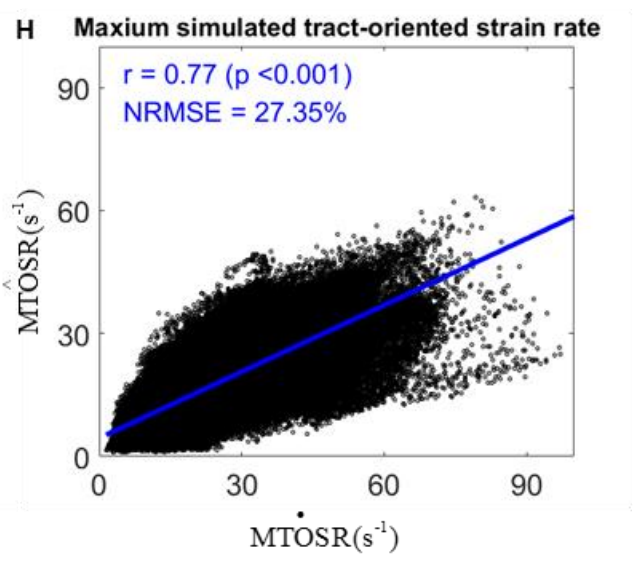

Figure 6. Comparison of computational principal strain rate and tract-oriented strain rate by both schemes. Upper row: Horizontal cross-sections of MPSR (A), MPSR (B), and their relative difference (C). Middle row: Horizontal cross-sections of MTOSR (D), MTOSR (E), and their relative difference (F). Lower row: Linear regression plots for element-wise principal strain rate in the whole brain $(G)$ and WM region $(H)$, respectively.

\section{Discussion}


The current study addressed the ambiguity of brain strain rate computation by presenting a theoretical clarification of two rival schemes, in which the strain rate was either computed as the time derivative of strain or derived from the strain-rate tensor. This theoretical disparity was further substantiated by discordant cross-scheme responses in experimental principal strain rate, and computational principal and tract-oriented strain rates. It was thus verified that strain rates were dependent on the specific computational schemes, underscoring that cross-scheme comparison of strain rate results was inappropriate. The newly calculated experimental strain rate curves, especially those with the peak values attained within the pulse durations, can be used to evaluate the strain rate response of FE models of human head, as exempfied for the ADAPT model in Appendix A.

Many in vivo, ex vivo, and in vitro models of TBI have reported a strain rate dependency of morphological or functional alterations of experimental tissues secondary to mechanical insults with precisely controlled loading parameters [6, 24, 60-70]. Several computational studies have also reported strain rate as an appropriate predictor for brain injury $[27,29,32,36,71]$. However, most TBI studies didn't report details of strain rate calculation $[8,21,22,71-74]$, making an appropriate interpretation of strain rate results impossible. For the handful of investigations with the strain rate calculation procedures elaborated, disparate inconsistency was noted in the computational scheme, posing inevitable challenges while comparing strain rate-related finding across studies. For example, LaPlaca and Thibault [24] established an in vitro TBI system to examine the neuronal responses to hydrodynamically-induced mechanical perturbations, in which the strain rate was computed by differentiating the strain with respect to time (i.e., scheme 1 ). This scheme was similarly adopted to derive the principal and/or tract-oriented strain rates from FE simulations of human brains $[27,35,38]$ and piglet brains $[29,32,36]$. In contrast, Carlsen, et al. [26] conducted a parametric study to evaluate the relationship between rotational head kinematics and brain tissue responses, in which the principal and tract-oriented strain rates were respectively derived as the first principal and tract-aligned components of the strain-rate tensor (i.e., scheme 2). Similarly, Patton, et al. [44] simulated 40 unhelmeted head impacts with known injury outcomes to evaluate the injury predictability of various 
metrics, of which the principal strain rate was computed as the maximum eigenvalue of strain-rate tensor (i.e., scheme 2). Our work demonstrated cross-scheme disparities in the resultant strain rate responses from the theoretical perspective as well as experimental and computational substantiation. These disparities highlighted that cross-scheme comparison of strain rate results is inappropriate. For example, the strain rate thresholds established by Patton, et al. [44] using scheme 2 were not comparable with the strain rate results derived by scheme 1 in the study by Hernandez and Camarillo [34], even though both studies used the exact same FE model [56]. Similarly, interpreting the FEderived strain rate outputs with reference to the experimentally determined strain rate thresholds computed by another scheme was also inappropriate. We recommended that, if applicable, clarifying the strain rate computational details be regarded as standard content in future studies.

As both schemes are in use for strain rate computation and no consensus has been reached within the research community, we thus computed the experimental strain rates from the cadaveric tests reported earlier [14] using both schemes. The newly calculated strain rate responses are then compared with values reported in other experimental studies (Table 1). When computing the strain rate as the time derivate of strain (i.e., scheme 1), the current experimental strain rate peaks largely exceeded those measured from the volunteer tests whose loading conditions were far from injury levels [75]. Sullivan, et al. [36] estimated the strain rate using the scheme 1 in six horizontally dissected piglet brains during rapid rotational events, in which the imposed angular velocities were one order of magnitude larger than those in [14]. Consequently, the reported strain rates in Sullivan, et al. [36] largely surpassed the current experimental results. When switching to scheme 2, Reese, et al. [42] reconstructed the velocity gradient from phase-contrast magnetic resonance imaging due to the vascular pulsatile and then computed 3D strain-rate tensor of brain parenchyma. This pulsatileinduced strain rate was two orders of magnitude smaller than the current experimental strain rates calculated by scheme 2 .

Table 1. Comparison of brain strain rates from existing studies. Note that the strain rate in Feng, et al. [75] was estimated from Figure 10 in the original article. 


\begin{tabular}{|c|c|c|c|c|}
\hline Scheme & Approach & Study & Loading conditions & Strain rate \\
\hline \multirow{3}{*}{$\begin{array}{l}\text { Scheme 1: } \\
\text { Time } \\
\text { derivative of } \\
\text { strain }\end{array}$} & Volunteer test & Feng, et al. [75] & $\begin{array}{l}\text { Linear acceleration: } 1.5 \mathrm{~g} \\
\text { Angular acceleration: } 120-140 \mathrm{rad} / \mathrm{s}^{2}\end{array}$ & $\begin{array}{l}\text { Peak principal strain } \\
\text { rate: } \sim 1.2-2.7 \mathrm{~s}^{-1}\end{array}$ \\
\hline & Animal test & $\begin{array}{l}\text { Sullivan, et al. } \\
\text { [36] }\end{array}$ & Angular velocity: $121 \pm 3.22 \mathrm{rad} / \mathrm{s}$ & $\begin{array}{l}\text { Mean principal strain } \\
\text { rate: } 150-189 \mathrm{~s}^{-1}\end{array}$ \\
\hline & PMHS test & Current study & $\begin{array}{l}\text { Linear acceleration: } 38-291 \mathrm{~g} \\
\text { Angular acceleration: } 2370-24206 \mathrm{rad} / \mathrm{s}^{2} \\
\text { Angular velocity: } 20.3 \pm 5.7 \mathrm{rad} / \mathrm{s}\end{array}$ & $\begin{array}{l}\text { Peak principal strain } \\
\text { rate: } 9.3-25.8 \mathrm{~s}^{-1}\end{array}$ \\
\hline \multirow{2}{*}{$\begin{array}{l}\text { Scheme 2: } \\
\text { Strain-rate } \\
\text { tensor }\end{array}$} & Volunteer test & $\begin{array}{l}\text { Reese, et al. } \\
{[42]}\end{array}$ & Vascular pulsatility & $\begin{array}{l}\text { Principal strain rate: } \\
0.1 \mathrm{~s}^{-1}\end{array}$ \\
\hline & PMHS test & Current study & $\begin{array}{l}\text { Linear acceleration: } 38-291 \mathrm{~g} \\
\text { Angular acceleration: } 2370-24206 \mathrm{rad} / \mathrm{s}^{2} \\
\text { Angular velocity: } 20.3 \pm 5.7 \mathrm{rad} / \mathrm{s}\end{array}$ & $\begin{array}{l}\text { Peak principal strain } \\
\text { rate: } 15.6-35.6 \mathrm{~s}^{-1}\end{array}$ \\
\hline
\end{tabular}

FE brain models are valuable instruments to explore the pathway from external mechanical insult and resultant brain trauma by linking FE-derived variables (e.g., strain rate) to recorded injury information. The reliability of such exploration can be partially guaranteed only if the FE-derived variables are validated against relevant experimental data. It has been long recognized that an FE head model validated against experimental pressure does not necessarily guarantee an acceptable predictability of the brain displacement or deformation [76-78]. More recently, several independent research groups advocated that a model to be used for strain prediction should be evaluated against the experimental brain strain, and not just the brain-skull relative motion $[15,28,79]$. When it comes to strain rate, there is only one FE model of piglet brain [36], to the best of the authors' knowledge, having the strain rate peaks (instead of the whole strain rate curves) evaluated against the experimental strain rates. As is the typical practice, FE head models validated against brain-skull relative motion and/or brain deformation are directly used for strain rate prediction. Although the strain rate can be derived from strain or displacement field via a chain of calculation (as detailed in section 2.1), a direct evaluation of FE-derived strain rate responses is preferred if the model is intended to be used for strain rate prediction. This is partially supported by the incongruent strain and strain rate validation performances of the FE model of piglet brain [36], in which the computational strain rate peaks exhibited superior correlation with their experimentally determined counterparts with respect to that between the experimental and computational strain peaks. Note that, in [36], the strain 
rate was computed as the time derivative of strain (i.e., scheme 1 in the current study). Taken together, to ensure the confidence of using FE head model for injury prediction based on strain rate, a direct evaluation of FE-derived stain rate response against its experimental counterpart (e.g., the newly calculated experimental strain rate curves in the current study) is preferred, as exemplified by the ADAPT model in Appendix A.

Several recent studies based on FE models of piglet brain $[29,32,36]$ have reported that the maximum tract-oriented strain rate exhibited superior injury predictability over maximum principal strain rate, while the majority of the existing FE investigations on human brain remain to solely scrutinize the maximum principal strain rate. We thus examined the relationship between the prevalently used principal strain rate and the recently advocated tract-oriented strain rate across all WM elements in the ADAPT model. As quantitatively illustrated in Figure 7, no consistent scaling from maximum principal strain rate to its tract-aligned counterpart was noted regardless of the computational scheme. This supports the potential incorporation of tract-oriented strain rate as an independent injury metric in future studies of human brain injury, in addition to the principal strain rate.

A Strain rates computed by scheme 1

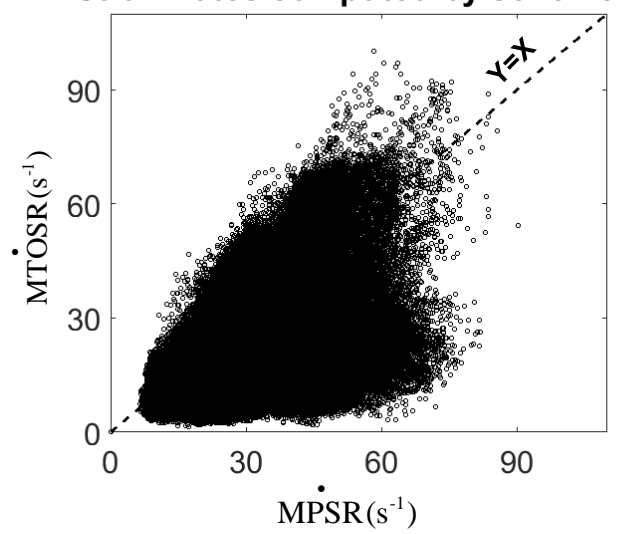

B Strain rates computed by scheme 2

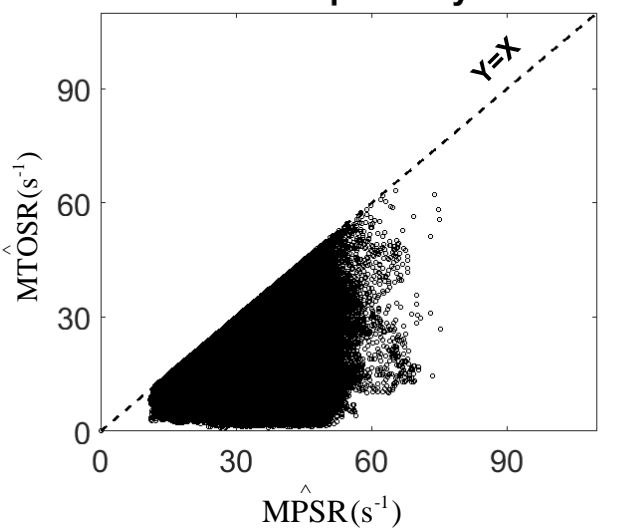

Figure 7. Element-wise comparisons of maximum principal strain rates and tract-related strain rates computed by scheme 1 (A) and scheme 2 (B) based on results of WM elements.

\subsection{Limitation and future work}

Several limitations in this study should be noted, which require further investigation. First, although our results clearly demonstrated cross-scheme disparities, we have no recommendation on 
which scheme is superior. This can be alternatively done by evaluating the injury predictability of these strain rate-based metrics computed by these two schemes. For the time being, choices may be prioritized differently depending on the intended purpose, but we strongly recommended that the exact strain rate computational schemes be clearly stated in future studies. Secondly, the cadaveric experiments analysed in this study were not without errors, as was acknowledged in the original studies $[14,80]$ and exhaustively evaluated in several recent independent studies $[15,28,79,81]$. Moreover, the principal strain rate responses estimated from the experimental data and the ADAPT model exhibited certain deviations (Appendix A), although this is the first FE human brain model performing strain rate evaluation. Even so, we'd like to highlight that the main advocation of the current work, i.e., cross-scheme comparison of the strain rate results was not valid, is theoretically supported by the content presented in the "Theoretical basis" section in the current study. Such a advocation should not be disapproved by these experimental and computational substantiations with certain deficits. Nevertheless, we acknowledge that further demonstration of cross-scheme disparity is deemed necessary by employing other experimental data and better validated FE models. Lastly, the current work focused on the disparity of strain rate as outputs. Concerning inconsistency was also noted in the constitutive modeling of strain rate-dependent material, in which the strain rate served as an input to describe the stress-strain relations of a given material [82].

\section{Conclusion}

Given the ambiguity and inconsistency of strain rate computational schemes in existing brain injury studies, the current work presented a theoretical clarification of two rival schemes: strain rates computed as the time derivative of strain and derived from the strain-rate tensor. Both schemes were employed to calculate experimental principal strain rate and computational principal and tractoriented strain rates, exhibiting highly scheme-dependent responses. Our work underscores that cross-scheme comparison of strain rate should be avoided and the exact strain rate computational 
scheme needs to be clarified in future studies. The newly calculated experimental strain rate data can be used to validate the strain rate response of FE models of human head.

\section{Acknowledgment}

This research has received funding from KTH Royal Institute of Technology (Stockholm, Sweden) and the Swedish Research Council (VR-2020-04496 and VR-2020-04724). The content of this article is solely the responsibility of the authors and does not necessarily represent the official views of funding agencies. The simulations were enabled by resources in project [SNIC 2021/5-406 and SNIC 2021/5459] provided by the Swedish National Infrastructure for Computing (SNIC) at the center for High Performance Computing (PDC), partially funded by the Swedish Research Council through grant agreement no. VR-2020-04496 and VR-2020-04724.

\section{Conflict of Interest}

The authors declare that they have no conflict of interest.

\section{References}

[1] D.K. Menon, K. Schwab, D.W. Wright, A.I. Maas, Position statement: definition of traumatic brain injury, Archives of physical medicine and rehabilitation, 91 (2010) 1637-1640.

[2] CDC, Centers for Disease Control and Prevention, National Center for Health Statistics: Mortality data on CDC WONDER, Available at: https://wonder.cdc.gov/mcd.html, (2020).

[3] M. Majdan, D. Plancikova, A. Brazinova, M. Rusnak, D. Nieboer, V. Feigin, A. Maas, Epidemiology of traumatic brain injuries in Europe: a cross-sectional analysis, The Lancet Public Health, 1 (2016) e76e83.

[4] B. Roozenbeek, A.I. Maas, D.K. Menon, Changing patterns in the epidemiology of traumatic brain injury, Nature Reviews Neurology, 9 (2013) 231-236.

[5] P.V. Bayly, E.H. Clayton, G.M. Genin, Quantitative imaging methods for the development and validation of brain biomechanics models, Annual review of biomedical engineering, 14 (2012) 369-396. [6] E. Bar-Kochba, M.T. Scimone, J.B. Estrada, C. Franck, Strain and rate-dependent neuronal injury in a 3D in vitro compression model of traumatic brain injury, Scientific Reports, 6 (2016) 1-11.

[7] A. Montanino, M. Saeedimasine, A. Villa, S. Kleiven, Axons Embedded in a Tissue May Withstand Larger Deformations Than Isolated Axons Before Mechanoporation Occurs, Journal of Biomechanical Engineering, 141 (2019).

[8] S. Kleiven, Predictors for traumatic brain injuries evaluated through accident reconstructions, Stapp car crash J, 51 (2007) 81-114.

[9] K. Bian, H. Mao, Mechanisms and variances of rotation-induced brain injury: a parametric investigation between head kinematics and brain strain, Biomechanics and modeling in mechanobiology, 19 (2020) 2323-2341. 
[10] E.G. Takhounts, S.A. Ridella, V. Hasija, R.E. Tannous, J.Q. Campbell, D. Malone, K. Danelson, J. Stitzel, S. Rowson, S. Duma, Investigation of Traumatic Brain Injuries Using the Next Generation of Simulated Injury Monitor (SIMon) Finite Element Head Model, Stapp car crash journal, 52 (2008) 1-31. [11] A.A. Weaver, K.A. Danelson, J.D. Stitzel, Modeling brain injury response for rotational velocities of varying directions and magnitudes, Annals of biomedical engineering, 40 (2012) 2005-2018.

[12] S. Ji, W. Zhao, J.C. Ford, J.G. Beckwith, R.P. Bolander, R.M. Greenwald, L.A. Flashman, K.D. Paulsen, T.W. McAllister, Group-wise evaluation and comparison of white matter fiber strain and maximum principal strain in sports-related concussion, Journal of neurotrauma, 32 (2015) 441-454.

[13] Z. Zhou, A.G. Domel, X. Li, G. Grant, S. Kleiven, D. Camarillo, M. Zeineh, White matter tractoriented deformation is dependent on real-time axonal fiber orientation, Journal of neurotrauma, 38 (2021) 1730-1745.

[14] W.N. Hardy, M.J. Mason, C.D. Foster, C.S. Shah, J.M. Kopacz, K.H. Yang, A.I. King, J. Bishop, M. Bey, W. Anderst, A Study of the Response of the Human Cadaver Head to Impact, Stapp Car Crash J, 51 (2007) 17-80.

[15] Z. Zhou, X. Li, S. Kleiven, W.N. Hardy, Brain strain from motion of sparse markers, Stapp car crash journal, 63 (2019) 1-27.

[16] A.K. Knutsen, A.D. Gomez, M. Gangolli, W.-T. Wang, D. Chan, Y.-C. Lu, E. Christoforou, J.L. Prince, P.V. Bayly, J.A. Butman, In vivo estimates of axonal stretch and 3D brain deformation during mild head impact, Brain multiphysics, 1 (2020) 100015.

[17] E.G. Takhounts, M.J. Craig, K. Moorhouse, J. McFadden, V. Hasija, Development of Brain Injury Criteria (BrIC), Stapp car crash journal, 57 (2013) 243-266.

[18] H. Mao, L. Zhang, B. Jiang, V.V. Genthikatti, X. Jin, F. Zhu, R. Makwana, A. Gill, G. Jandir, A. Singh, Development of a finite element human head model partially validated with thirty five experimental cases, Journal of biomechanical engineering, 135 (2013) 111002.

[19] T. Laituri, S. Henry, K. Pline, G. Li, M. Frankstein, P. Weerappuli, New Risk Curves for NHTSA's Brain Injury Criterion (BrIC): Derivations and Assessments, Stapp car Crash Journal, 60 (2016) 301-362.

[20] Z. Zhou, X. Li, Y. Liu, M. Fahlstedt, M. Georgiadis, X. Zhan, S.J. Raymond, G. Grant, S. Kleiven, D. Camarillo, Towards a comprehensive delineation of white matter tract-related deformation, bioRxiv, (2021).

[21] J.G. Beckwith, W. Zhao, S. Ji, A.G. Ajamil, R.P. Bolander, J.J. Chu, T.W. McAllister, J.J. Crisco, S.M. Duma, S. Rowson, Estimated brain tissue response following impacts associated with and without diagnosed concussion, Annals of biomedical engineering, 46 (2018) 819-830.

[22] A. Post, M. Kendall, D. Koncan, J. Cournoyer, T.B. Hoshizaki, M.D. Gilchrist, S. Brien, M.D. Cusimano, S. Marshall, Characterization of persistent concussive syndrome using injury reconstruction and finite element modelling, Journal of the mechanical behavior of biomedical materials, 41 (2015) 325-335.

[23] B. Rashid, M. Destrade, M.D. Gilchrist, Influence of preservation temperature on the measured mechanical properties of brain tissue, Journal of biomechanics, 46 (2013) 1276-1281.

[24] M.C. LaPlaca, L.E. Thibault, An in vitro traumatic injury model to examine the response of neurons to a hydrodynamically-induced deformation, Annals of biomedical engineering, 25 (1997) 665-677.

[25] Z. Zhou, X. Li, A.G. Domel, E.L. Dennis, M. Georgiadis, Y. Liu, S.J. Raymond, G. Grant, S. Kleiven, D. Camarillo, M. Zeineh, The Presence of the Temporal Horn Exacerbates the Vulnerability of Hippocampus during Head Impacts Frontiers in bioengineering and biotechnology, (2022) In press, DOI: $10.3389 /$ fbioe.2022.754344.

[26] R.W. Carlsen, A.L. Fawzi, Y. Wan, H. Kesari, C. Franck, A quantitative relationship between rotational head kinematics and brain tissue strain from a 2-D parametric finite element analysis, Brain Multiphysics, 2 (2021) 100024.

[27] A.I. King, K.H. Yang, L. Zhang, W. Hardy, D.C. Viano, Is head injury caused by linear or angular acceleration, in: IRCOBI conference, Lisbon, Portugal, 2003.

[28] Z. Zhou, X. Li, S. Kleiven, C.S. Shah, W.N. Hardy, A Reanalysis of Experimental Brain Strain Data: Implication for Finite Element Head Model Validation, Stapp car crash journal, 62 (2018) 293-318. 
[29] M. Hajiaghamemar, T. Wu, M.B. Panzer, S.S. Margulies, Embedded axonal fiber tracts improve finite element model predictions of traumatic brain injury, Biomechanics and modeling in mechanobiology, 19 (2020) 1109-1130.

[30] F. Hernandez, C. Giordano, M. Goubran, S. Parivash, G. Grant, M. Zeineh, D. Camarillo, Lateral impacts correlate with falx cerebri displacement and corpus callosum trauma in sports-related concussions, Biomechanics and modeling in mechanobiology, 18 (2019) 631-649.

[31] L.E. Miller, J.E. Urban, E.M. Davenport, A.K. Powers, C.T. Whitlow, J.A. Maldjian, J.D. Stitzel, Brain strain: computational model-based metrics for head impact exposure and injury correlation, Annals of biomedical engineering, 49 (2021) 1083-1096.

[32] M. Hajiaghamemar, S.S. Margulies, Multi-scale white matter tract embedded brain finite element model predicts the location of traumatic diffuse axonal injury, Journal of Neurotrauma, 38 (2021) 144157.

[33] M. Hajiaghamemar, M. Seidi, S.S. Margulies, Head rotational kinematics, tissue deformations, and their relationships to the acute traumatic axonal injury, Journal of biomechanical engineering, 142 (2020) 031006.

[34] F. Hernandez, D.B. Camarillo, Voluntary head rotational velocity and implications for brain injury risk metrics, Journal of neurotrauma, 36 (2019) 1125-1135.

[35] D. Sahoo, C. Deck, R. Willinger, Brain injury tolerance limit based on computation of axonal strain, Accident Analysis \& Prevention, 92 (2016) 53-70.

[36] S. Sullivan, S.A. Eucker, D. Gabrieli, C. Bradfield, B. Coats, M.R. Maltese, J. Lee, C. Smith, S.S. Margulies, White matter tract-oriented deformation predicts traumatic axonal brain injury and reveals rotational direction-specific vulnerabilities, Biomechanics and modeling in mechanobiology, 14 (2015) 877-896.

[37] E.W. Vogel, M.B. Panzer, F.N. Morales, N. Varghese, C.R. Bass, D.F. Meaney, B. Morrison, Direct observation of low strain, high rate deformation of cultured brain tissue during primary blast, Annals of biomedical engineering, 48 (2020) 1196-1206.

[38] T. Wu, M. Hajiaghamemar, J.S. Giudice, A. Alshareef, S.S. Margulies, M.B. Panzer, Evaluation of tissue-level brain injury metrics using species-specific simulations, Journal of neurotrauma, (2021).

[39] L. Zhang, K.H. Yang, A.I. King, D.C. Viano, A new biomechanical predictor for mild traumatic brain injury-A preliminary finding, in: Proc. 2003 Summer Bioengineering Conference, 2003, pp. 137-138.

[40] A.G. Holzapfel, Nonlinear solid mechanics: A continuum approach for engineering, Wiley, Chichester, 2000.

[41] R. Terpsma, R.W. Carlsen, R. Szalkowski, S. Malave, A.L. Fawzi, C. Franck, C. Hovey, Head Impact Modeling to Support a Rotational Combat Helmet Drop Test, Military Medicine, (2021).

[42] T.G. Reese, D.A. Feinberg, J. Dou, V.J. Wedeen, Phase contrast MRI of myocardial 3D strain by encoding contiguous slices in a single shot, Magnetic Resonance in Medicine: An Official Journal of the International Society for Magnetic Resonance in Medicine, 47 (2002) 665-676.

[43] X.G. Tan, V.S.S.S. Sajja, M.M. D'Souza, R.K. Gupta, J.B. Long, A.K. Singh, A. Bagchi, A Methodology to Compare Biomechanical Simulations With Clinical Brain Imaging Analysis Utilizing Two Blunt Impact Cases, Frontiers in Bioengineering and Biotechnology, 9 (2021).

[44] D.A. Patton, A.S. McIntosh, S. Kleiven, The biomechanical determinants of concussion: finite element simulations to investigate tissue-level predictors of injury during sporting impacts to the unprotected head, Journal of applied biomechanics, 31 (2015) 264-268.

[45] V. Malis, U. Sinha, S. Sinha, 3D Muscle Deformation Mapping at Submaximal Isometric Contractions: Applications to Aging Muscle, Frontiers in physiology, 11 (2020) 1563.

[46] V. Mazzoli, L.M. Gottwald, E.S. Peper, M. Froeling, B.F. Coolen, N. Verdonschot, A.M. Sprengers, P. van Ooij, G.J. Strijkers, A.J. Nederveen, Accelerated $4 \mathrm{D}$ phase contrast MRI in skeletal muscle contraction, Magnetic resonance in medicine, 80 (2018) 1799-1811.

[47] U. Sinha, V. Malis, J.-S. Chen, R. Csapo, R. Kinugasa, M.V. Narici, S. Sinha, Role of the Extracellular Matrix in Loss of Muscle Force With Age and Unloading Using Magnetic Resonance Imaging, Biochemical Analysis, and Computational Models, Frontiers in Physiology, 11 (2020) 626. 
[48] U. Sinha, V. Malis, R. Csapo, M. Narici, S. Sinha, Shear strain rate from phase contrast velocity encoded MRI: application to study effects of aging in the medial gastrocnemius muscle, Journal of Magnetic Resonance Imaging, 48 (2018) 1351-1357.

[49] S.M. Felton, T.A. Gaige, T. Benner, R. Wang, T.G. Reese, V.J. Wedeen, R.J. Gilbert, Associating the mesoscale fiber organization of the tongue with local strain rate during swallowing, Journal of biomechanics, 41 (2008) 1782-1789.

[50] S.M. Felton, T.A. Gaige, T.G. Reese, V.J. Wedeen, R.J. Gilbert, Mechanical basis for lingual deformation during the propulsive phase of swallowing as determined by phase-contrast magnetic resonance imaging, Journal of Applied Physiology, 103 (2007) 255-265.

[51] J. Dou, W.Y.I. Tseng, T.G. Reese, V.J. Wedeen, Combined diffusion and strain MRI reveals structure and function of human myocardial laminar sheets in vivo, Magnetic Resonance in Medicine: An Official Journal of the International Society for Magnetic Resonance in Medicine, 50 (2003) 107-113.

[52] V.J. Wedeen, Magnetic resonance imaging of myocardial kinematics. Technique to detect, localize, and quantify the strain rates of the active human myocardium, Magnetic resonance in medicine, 27 (1992) 52-67.

[53] M.D. Robson, R.T. Constable, Three - dimensional strain - rate imaging, Magnetic resonance in medicine, 36 (1996) 537-546.

[54] R. de Rooij, E. Kuhl, Constitutive modeling of brain tissue: current perspectives, Applied Mechanics Reviews, 68 (2016).

[55] X. Li, Z. Zhou, S. Kleiven, An anatomically detailed and personalizable head injury model: Significance of brain and white matter tract morphological variability on strain, Biomechanics and modeling in mechanobiology, 20 (2021) 403-431.

[56] S. Kleiven, Predictors for traumatic brain injuries evaluated through accident reconstructions, Stapp car crash journal, 51 (2007) 81-114.

[57] A.M. Nahum, R. Smith, C.C. Ward, Intracranial pressure dynamics during head impact, in: Proceedings of the 21st Stapp Car Crash Conference, SAE Technical Paper, Warrendale, PA, USA, 1977, pp. 337-366.

[58] S. Mori, K. Oishi, H. Jiang, L. Jiang, X. Li, K. Akhter, K. Hua, A.V. Faria, A. Mahmood, R. Woods, Stereotaxic white matter atlas based on diffusion tensor imaging in an ICBM template, Neuroimage, 40 (2008) 570-582.

[59] F. Hernandez, L.C. Wu, M.C. Yip, K. Laksari, A.R. Hoffman, J.R. Lopez, G.A. Grant, S. Kleiven, D.B. Camarillo, Six degree-of-freedom measurements of human mild traumatic brain injury, Annals of biomedical engineering, 43 (2015) 1918-1934.

[60] D.K. Cullen, M.C. LaPlaca, Neuronal response to high rate shear deformation depends on heterogeneity of the local strain field, Journal of neurotrauma, 23 (2006) 1304-1319.

[61] D.K. Cullen, C.M. Simon, M.C. LaPlaca, Strain rate-dependent induction of reactive astrogliosis and cell death in three-dimensional neuronal-astrocytic co-cultures, Brain research, 1158 (2007) 103-115.

[62] D.K. Cullen, V.N. Vernekar, M.C. LaPlaca, Trauma-induced plasmalemma disruptions in threedimensional neural cultures are dependent on strain modality and rate, Journal of neurotrauma, 28 (2011) 2219-2233.

[63] B. Elkin, B. Morrison III, Region-specific tolerance criteria for the living brain, Stapp car Crash Journal, 51 (2007) 127-138.

[64] D.M. Geddes, R.S. Cargill, M.C. LaPlaca, Mechanical stretch to neurons results in a strain rate and magnitude-dependent increase in plasma membrane permeability, Journal of neurotrauma, 20 (2003) 1039-1049.

[65] M.C. LaPlaca, D.K. Cullen, J.J. McLoughlin, R.S. Cargill II, High rate shear strain of three-dimensional neural cell cultures: a new in vitro traumatic brain injury model, Journal of biomechanics, 38 (2005) 1093-1105.

[66] M.C. LaPlaca, V.M.-Y. LEE, L.E. THIBAULT, An in vitro model of traumatic neuronal injury: loading rate-dependent changes in acute cytosolic calcium and lactate dehydrogenase release, Journal of neurotrauma, 14 (1997) 355-368. 
[67] Y. Li, C. Li, C. Gan, K. Zhao, J. Chen, J. Song, T. Lei, A precise, controllable in vitro model for diffuse axonal injury through uniaxial stretch injury, Frontiers in neuroscience, 13 (2019) 1063.

[68] H. Nakadate, E. Kurtoglu, H. Furukawa, S. Oikawa, S. Aomura, A. Kakuta, Y. Matsui, Strain-rate dependency of axonal tolerance for uniaxial stretching, in, SAE Technical Paper, 2017.

[69] R. Shi, J. Whitebone, Conduction deficits and membrane disruption of spinal cord axons as a function of magnitude and rate of strain, Journal of neurophysiology, 95 (2006) 3384-3390.

[70] A. Singh, S. Kallakuri, C. Chen, J.M. Cavanaugh, Structural and functional changes in nerve roots due to tension at various strains and strain rates: an in-vivo study, Journal of neurotrauma, 26 (2009) 627-640.

[71] T.W. McAllister, J.C. Ford, S. Ji, J.G. Beckwith, L.A. Flashman, K. Paulsen, R.M. Greenwald, Maximum principal strain and strain rate associated with concussion diagnosis correlates with changes in corpus callosum white matter indices, Annals of biomedical engineering, 40 (2012) 127-140.

[72] Y. Liu, A.G. Domel, N.J. Cecchi, E. Rice, A.A. Callan, S.J. Raymond, Z. Zhou, X. Zhan, Y. Li, M.M. Zeineh, G.A. Grant, D.B. Camarillo, Time Window of Head Impact Kinematics Measurement for Calculation of Brain Strain and Strain Rate in American Football, Annals of Biomedical Engineering, 49 (2021) 2791-2804.

[73] H. Mao, L. Zhang, K. Yang, A. King, Application of a finite element model of the brain to study traumatic brain injury mechanisms in the rat, Stapp car Crash Journal, 50 (2006) 583-600.

[74] D.C. Viano, I.R. Casson, E.J. Pellman, L. Zhang, A.I. King, K.H. Yang, Concussion in professional football: brain responses by finite element analysis: part 9, Neurosurgery, 57 (2005) 891-916.

[75] Y. Feng, T.M. Abney, R.J. Okamoto, R.B. Pless, G.M. Genin, P.V. Bayly, Relative brain displacement and deformation during constrained mild frontal head impact, Journal of the Royal Society Interface, 7 (2010) 1677-1688.

[76] D. Bradshaw, C. Morfrey, Pressure and shear responses in brain injury models, in: Proceedings: International Technical Conference on the Enhanced Safety of Vehicles, National Highway Traffic Safety Administration, 2001, pp. 10 p.-10 p.

[77] S. Kleiven, W. Hardy, Correlation of an FE Model of the Human Head with Local Brain Motion-Consequences for Injury Prediction, Stapp car Crash Journal, 46 (2002) 123-144.

[78] W. Zhao, S. Ruan, S. Ji, Brain pressure responses in translational head impact: a dimensional analysis and a further computational study, Biomechanics and modeling in mechanobiology, 14 (2015) 753-766.

[79] W. Zhao, S. Ji, Displacement-and strain-based discrimination of head injury models across a wide range of blunt conditions, Annals of biomedical engineering, 48 (2020) 1661-1677.

[80] W.N. Hardy, C.D. Foster, M.J. Mason, K.H. Yang, A.I. King, S. Tashman, Investigation of head injury mechanisms using neutral density technology and high-speed biplanar X-ray, Stapp car crash journal, 45 (2001) 337-368.

[81] S. Dutrisac, M. Brannen, T.B. Hoshizaki, H. Frei, O. Petel, A parametric analysis of embedded tissue marker properties and their effect on the accuracy of displacement measurements, Journal of Biomechanical Engineering, (2021).

[82] M. Destrade, G. Saccomandi, M. Vianello, Proper formulation of viscous dissipation for nonlinear waves in solids, The Journal of the Acoustical Society of America, 133 (2013) 1255-1259.

[83] C. Gehre, H. Gades, P. Wernicke, Objective rating of signals using test and simulation responses, in: 21st International Technical Conference on the Enhanced Safety of Vehicles Conference (ESV), 2009, pp. 09-0407.

\section{Appendix A: Strain rate evaluation of finite element head model}


Strain rate responses of the ADAPT model were validated against the newly calculated experimental results. Three representative impacts were selected, including C1 in C288T3 (sagittal impact), C1 in C380T2 (horizontal impact), and C1 in C380T4 (coronal impact). To better reflect the cranial geometry of experimental specimens, the model was scaled to match the reported anthropometric measurements of the cadaveric heads. The nodes nearest to the initial position of the experimental NDT markers were identified and further informed the formulation of the eight tetrahedral elements representing the tissue encased by the experimental NDT cluster. Following the same approach of computing experimental strain rate, the 3D motion of the identified nodes with respect to the skull was acquired from the whole head model simulation and further exercised the tetrahedral elements, through which the first principal strain rate of each element was respectively extracted as the time derivatives of the first principal strain (i.e., $\operatorname{PSR}(t)$ for the scheme 1 ) and maximum eigenvalue of the strain-rate tensor (i.e., $\hat{\operatorname{PSR}}(t)$ for the scheme 2 ). For each cluster, the strain rates from all eight elements were averaged.

The correlation between the computationally predicted strain rate responses and experimentally determined measurements was quantitatively evaluated by the cross-correlation method in CORA (correlation and analysis) with technical details available in [83]. Briefly, the crosscorrelation method in CORA scored the level of correlation between a pair of time history curves by equally weighting their similarities in shape, size, and phase. The CORA score ranges from 0 (no correlation) to 1 (perfect match). The exact setting of relevant parameters for CORA implementation and biofidelity classification based on CORA scores are respectively presented in Table A1 and Table A2.

Table A1. Parameter setting for the cross-correlation in CORA.

\begin{tabular}{ll|ll}
\hline Parameter & Value & Parameter & Value \\
\hline A_THRES & 0.030 & INT_MIN & 0.70 \\
B_THRES & 0.075 & K_V & 3 \\
A_VAL & 0.010 & K_P & 1
\end{tabular}




\begin{tabular}{ll|ll} 
B_DELTA_END & 0.200 & K_G & 1 \\
T_MIN / T_MAX (ms) & $0 / 40$ & G_V & 0.333 \\
D_MIN & 0.01 & G_P & 0.333 \\
D_MAX & 0.25 & G_G & 0.333
\end{tabular}

Table A2. Biofidelity rate and corresponding range of CORA score defined by ISO/TR 9790.

\begin{tabular}{lc}
\hline Biofidelity rating & CORA score \\
\hline Excellent & $0.86-1.00$ \\
Good & $0.65-0.86$ \\
Fair & $0.44-0.65$ \\
Margin & $0.26-0.44$ \\
Unacceptable & $0-0.26$ \\
\hline
\end{tabular}

Figure A1 illustrates the comparison of model-predicted strain rate curves against the experiment-derived results for both schemes. The CORA score ranged from 0.65 to 0.71 for scheme 1 and from 0.64 to 0.94 for scheme 2 . According to the biofidelity classification based on CORA scores (Table A2), the strain rate response of the ADAPT model can be rated as "good" for scheme 1, and "good" to "excellent" for scheme 2.
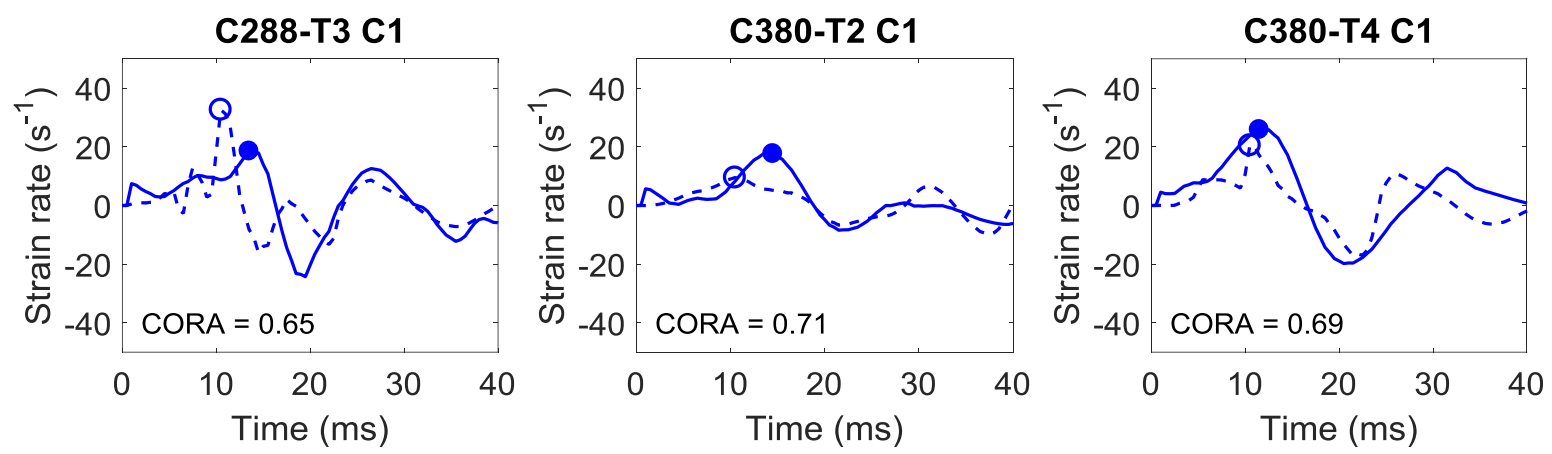

-Experimental principal strain rate (scheme 1) - Simulated principal strain rate (scheme 1)

C288-T3 C1

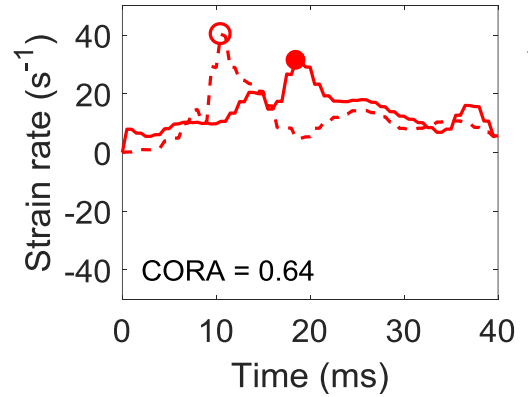

C380-T2 C1

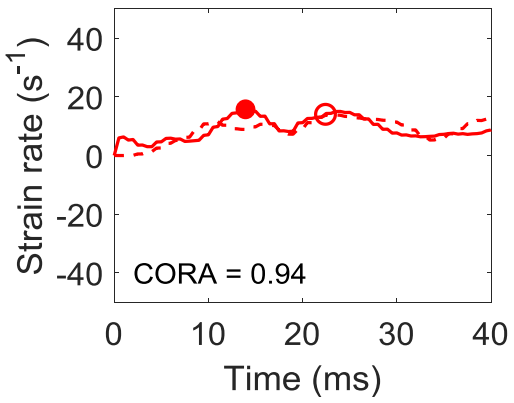

C380-T4 C1

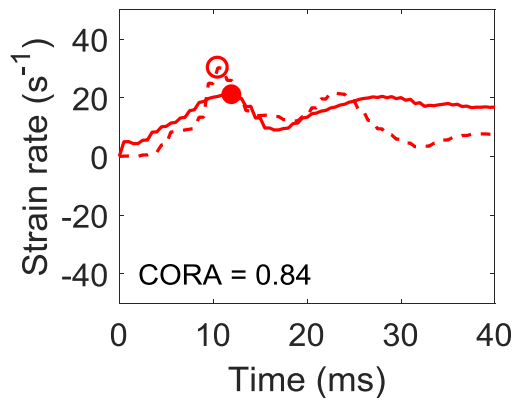

- Experimental principal strain rate (scheme 2) - - Simulated principal strain rate (scheme 2) 
bioRxiv preprint doi: https://doi.org/10.1101/2022.02.04.478773; this version posted February 8, 2022. The copyright holder for this preprint (which was not certified by peer review) is the author/funder. All rights reserved. No reuse allowed without permission.

Figure A1. Comparison of experimental and simulated strain rates computed by scheme 1 (upper row) and scheme 2 (low row). 